\title{
Brain stem and cerebellum volumetric analysis of Machado Joseph disease patients
}

\author{
Sarah Teixeira Camargos, Wilson Marques-Jr., Antonio Carlos dos Santos
}

\begin{abstract}
Machado-Joseph disease, or spinocerebellar ataxia type 3(MJD/SCA3), is the most frequent late onset spinocerebellar ataxia and results from a CAG repeat expansion in the ataxin-3 gene. Previous studies have found correlation between atrophy of cerebellum and brainstem with age and CAG repeats, although no such correlation has been found with disease duration and clinical manifestations. In this study we test the hypothesis that atrophy of cerebellum and brainstem in MJD/SCA3 is related to clinical severity, disease duration and CAG repeat length as well as to other variables such as age and ICARS (International Cooperative Ataxia Rating Scale). Whole brain high resolution MRI and volumetric measurement with cranial volume normalization were obtained from $15 \mathrm{MJD} /$ SCA3 patients and 15 normal, age and sex-matched controls. We applied ICARS and compared the score with volumes and CAG number, disease duration and age. We found significant correlation of both brain stem and cerebellar atrophy with CAG repeat length, age, disease duration and degree of disability. The Spearman rank correlation was stronger with volumetric reduction of the cerebellum than with brain stem. Our data allow us to conclude that volumetric analysis might reveal progressive degeneration after disease onset, which in turn is linked to both age and number of CAG repeat expansions in SCA 3. Key words: CAG expansion, Machado-Joseph disease, MJD/SCA3, spinocerebellar ataxia, volumetry.
\end{abstract}

Análise volumétrica de cerebelo e tronco cerebral de pacientes com doença de Machado Joseph

\section{RESUMO}

Doença de Machado-Joseph, ou ataxia espinocerebelar tipo 3 (MJD/SCA3) é ataxia espinocerebelar de início tardio mais frequente e resulta de uma expansão da repetição CAG no gene da ataxina-3. Estudos precedentes encontraram correlação entre a atrofia do cerebelo e do tronco cerebral com a idade e número de expansões CAG. Tais correlações não foram encontradas em relação ao tempo de doença ou manifestações clínicas. Neste estudo testamos a hipótese de que a atrofia do cerebelo e do tronco encefálico em MJD/SCA3 está relacionada à gravidade clínica, duração da doença e

\section{Correspondence}

Antonio Carlos dos Santos Rua Tenente Catão Roxo 1076 14051-140 Ribeirão Preto SP - Brasil E-mail- acsantos@fmrp.usp.br

\section{Support \\ This work was supported by FAEPA (Fundação de Amparo ao Ensino e Pesquisa do Hospital das Clínicas da Faculdade de Medicina de Ribeirão Preto), CAPES (Coordenação de Aperfeiçoamento de Pessoal de Nível Superior) and FAPESP (Fundação de Amparo a Pesquisa do Estado de São Paulo)}

Received 1 September 2010 Received in final form 23 November 2010 Accepted 30 November 2010 número de repetições CAG, bem como com outras variáveis como a idade e a ICARS (escala cooperativa internacional de avaliação de ataxias). Foram realizados estudos de imagem pela ressonância magnética de alta resolução e volumetria com normalização de volume craniano de 15 pacientes portadores de MJD/SCA3 e 15 controles pareados por idade e sexo. Nós aplicamos a ICARS e correlacionamos com o escore de volumes e número de CAG, duração da doença e idade. Encontramos correlação significativa entre atrofia de tronco cerebral e cerebelo com duração da doença, repetição CAG, idade e grau de acometimento da doença. O índice de correlação de Spearman foi maior em relação à atrofia de cerebelo do que à atrofia de tronco. Nossos dados permitem concluir que a análise volumétrica pode revelar degeneração progressiva após o início da doença que, por sua vez, está ligada à idade e número de expansões CAG em SCA 3.

Palavras-chave: expansão CAG, doença de Machado-Joseph, MJD/SCA3, ataxia espino cerebelar, volumetria.

Departamento de Neurologia. Faculdade de Medicina de Ribeirão Preto, Universidade de São Paulo, Ribeirão Preto SP, Brazil. 
The autosomal dominant spinocerebellar ataxias (SCA) constitute a large and heterogeneous group of late-onset diseases. To date, at least 29 of them have been mapped, and 15 genes have been identified. An exonic unstable CAG repeat expansion is the most frequent mutation associated with this group of neurodegenerative disorders ${ }^{1}$.

Machado Joseph disease (MJD) or spinocerebellar ataxia type 3 (MJD/SCA3) seems to be the most common late-onset spinocerebellar ataxia in many world regions, including Germany, Brazil, Portugal, India, France, North America, Japan, China and Taiwan ${ }^{1-5}$. In normal individuals, the gene contains between 12 and 44 CAG repeats, whereas most patients have between 61 to 87 CAG repeats 6 .

This disease has been consistently associated to the presence of cerebellar atrophy that was initially indirectly demonstrated by pneumoencephalography ${ }^{7}$. More recently, qualitative and quantitative magnetic resonance image (MRI) techniques provide a morphologic evaluation of the brain structures involved in this and other central nervous system diseases (CNS), allowing to study the progression of the abnormalities and possible correlations between gene defects, morphological changes and clinical phenotypes, leading to a better understanding of these diseases and their natural history ${ }^{8,9}$.

Since the identification of the Ataxin 3 gene, several of such studies have been undertaken in MJD/SCA3. Some authors found a moderate olivary and cerebellar atrophy ${ }^{10}$, while others reported that cerebellar and brain stem atrophy are significantly more severe in SCA1 and SCA2, proposing that SCA2 and MJD/SCA3 could be distinguished by studying the velocity of the saccadic ocular movement and the diameter of the middle cerebellar peduncle $^{11}$. Some authors reported that atrophy was age and disease-duration dependent, affecting moderately the cerebellum and more severely the brainstem, specially the pontine tegmentum ${ }^{12}$. These findings were not confirmed by others that found correlation with age at examination and CAG repeats but not with disease duration and clinical severity ${ }^{8}$. This divergence increased with another study, which failed to find correlation between CAG expansion and atrophy in a series of SCA1, SCA2 and SCA3 patients ${ }^{13}$. Cerebellar and pontine volume seems to be more involved in SCA7 than in MJD/SCA3 and SCA6, regardless of severity of symptoms or duration of the disease ${ }^{14}$.

More recently, the interest have focused on pons and basal ganglia involvement. Interestingly, a study showed that the atrophic process is not uniform inside the pons, being the tegmentum more affected than the pontine base ${ }^{9}$.

In this study we tried to sort out the correlations be- tween image findings with clinical and genetic data applying volumetric MRI measurements of posterior fossa structures, aiming to more precisely define the link between morphology and function in MJD/SCA3.

\section{METHOD}

\section{Subjects}

Fifteen consecutive patients with molecularly proven MJD/SCA3, and fifteen healthy normal controls matched for gender and age, were studied after excluding those with history of alcohol abuse and any other systemic or neurological disease. Disease duration was measured as years elapsed since the first motor symptom, and disease severity was measured with the International Cooperative Ataxia Rating Scale (ICARS) ${ }^{15}$. This study was approved by the Research Ethics Committee of our institution, and all patients signed an informed consent.

\section{Molecular analysis}

Lymphocyte DNA was isolated according to routine technique. The MJD/SCA3 gene was amplified using PCR as described before ${ }^{6}$. PCR product was submitted to $4 \%$ polyacrylamide gel electrophoresis in an automatic gene sequencer (ABI PRISM 377 DNA SEQUENCERUSA). Peak product was analyzed in relation to base pair number. CAG expansion number was calculated according to the formula: (number of base pairs- 160)/3.

\section{Image acquisition and processing}

MRI was performed on a 1.5-T system (Magneton Vision, Siemens, Erlanger, Germany). The study protocol included a fast-spin echo axial, double echo PD-T2weighted and axial FLAIR sequence to screen for focal lesions, and T1-weighted, three-dimensional spoiled gradient-recalled acquisition sequence with $1 \mathrm{~mm}$ slicethickness for volumetric measurements.

\section{Segmentation and normalization}

A trained operator (STC) blinded about the clinical data delineated the region-of-interest (ROI) manually based on axial, coronal and sagittal images, according to anatomic landmarks already described ${ }^{16}$ (Figure). Volumetry was performed based on the whole brain gradient echo 3D, with $1 \mathrm{~mm}^{3}$ isotropic voxel, using the Display software package (Brain Image Center, Montreal Neurological Institute, McGill University). A semi-automatic algorithm with threshold of signal intensity was used to detect the interface between CSF, gray and white matter, in order to minimize the partial volume artifact. Volumes were obtained by voxel quantification of the brain stem and cerebellum. The former was divided into midbrain, pons and medulla oblongata, while the latter was divided into vermis regions (V1, V2 e V3), tonsils and hemi- 

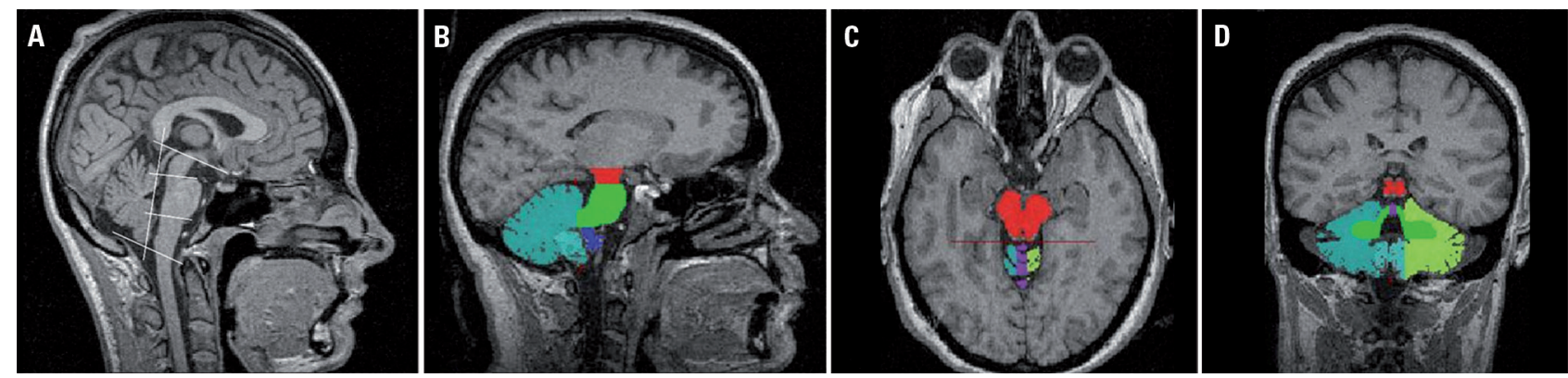

Figure. The segmentation of the posterior fossa structures were done according the color labels showed in [B] (sagittal), [C] (axial), and [D] (coronal) images, after brain stem division according the boundaries showed in [A].

Table 1. Brain stem and cerebellum volumes after normalization.

\begin{tabular}{|c|c|c|c|c|}
\hline Structure & Control (Mean $\pm S D)$ & Patient (Mean \pm SD) & Variation (\%) & $p$ \\
\hline Mesencephalon & $3.765 \pm 0.386$ & $3.364 \pm 0.437$ & 10.6 & $<0.05$ \\
\hline Pons & $13.408 \pm 2.007$ & $8.614 \pm 1.935$ & 35.7 & $<0.01$ \\
\hline Medulla oblongata & $2.618 \pm 0.896$ & $2.105 \pm 0.304$ & 19.6 & $<0.05$ \\
\hline Total brain stem & $19.792 \pm 2.672$ & $14.152 \pm 2.355$ & 28.5 & $<0.01$ \\
\hline Tonsil & $2.471 \pm 0.772$ & $2.512 \pm 0.856$ & - & $>0.05$ \\
\hline V1 & $1.515 \pm 0.302$ & $0.969 \pm 0.2009$ & 35.7 & $<0.01$ \\
\hline V2 & $0.878 \pm 0.108$ & $0.554 \pm 0.171$ & 36.9 & $<0.01$ \\
\hline V3 & $1.130 \pm 0.181$ & $0.867 \pm 0.205$ & 23.8 & $<0.05$ \\
\hline Total vermis & $3.525 \pm 0.544$ & $2.391 \pm 0.509$ & 32.2 & $<0.01$ \\
\hline L-hemisphere & $33.080 \pm 2.861$ & $25.635 \pm 4.1025$ & 22.5 & $<0.05$ \\
\hline R-hemisphere & $30.599 \pm 8.640$ & $26.105 \pm 4.610$ & 14.7 & $<0.05$ \\
\hline Total cerebellum & $69.677 \pm 10.012$ & $56.644 \pm 9.602$ & 18.7 & $<0.05$ \\
\hline
\end{tabular}

spheres. We used intracranial volume (ICV) including brain and cerebrospinal fluid for normalization of volumetric data, as previously published ${ }^{17}$. The operator was blind in relation to the identity of control and patients.

\section{Statistical analysis}

Group comparisons were performed with the Mann Whitney U test allowing a confidence interval of at least $95 \%$. Correlation of clinical findings and volumes were analyzed using Spearman's bi-variate correlation. The software package SPSS for Windows (SPSS Inc., Ilinois; version 8.0) was used.

\section{RESULTS}

Patients age ranged from 18 to 72 years old (mean $44.3 \pm 15.6$ ), and the the male: female ratio was 8:7. Disease duration ranged from 1 to 21 years (mean $8.7 \pm 5.81$ ) and CAG number from 65 to 88 repeats $(72.7 \pm 5.3)$.

Except for tonsils, all measured structures were significantly reduced in patients than in controls (Table 1). Correcting the acquired values for the total intracranial volume did not change significantly the findings. The total brain stem reduction observed in the patient group was $28.5 \%$, and the most affected brain stem structure was pons, with an average volume reduction of $35.7 \%$. The total cerebellum volume reduction was $18.7 \%$, mainly due to vermis (32.2\%) and hemispheres (22.5 and $14.7 \%$ ).

As shown in Table 2, an inverse correlation of disease duration were found with cerebellar hemispheres $(-0.7$, $-0.56 ; \mathrm{p}>0.05)$, total cerebellum $(-0.7 ; \mathrm{p}<0.05), \mathrm{V} 2(-0.69$ $\mathrm{p}<0.05)$ and mesencephalon volumes $(-0.52 ; \mathrm{p}>0.05)$. Another inverse correlation was found with CAG repeat number and total vermis $(-0.7, \mathrm{p}>0.05), \mathrm{V} 1(-0.69$, $\mathrm{p}>0.05)$ and mesencephalon volumes $(-0.53, \mathrm{p}>0.05)$. With the exception of tonsils, age was inversely correlated with all cerebellar structures. ICARS had an inverse correlation with the volumes of pons $(-0.68, p>0.05)$, total brain stem $(-0.62, \mathrm{p}>0.05), \mathrm{V} 2(-0.52, \mathrm{p}>0.05)$, cerebellar hemispheres $(-0.63 ;-0.49, \mathrm{p}>0.05)$ and total cerebellum $(-0.6, p>0.05)$. In addition, ICARS and disease duration showed a direct correlation. No correlations were found between studied parameters and medulla oblongata and tonsil.

\section{DISCUSSION}

In this study, we tried to correlate a volumetric analysis based on MRI examination with clinical and genetic data, to better understand the natural history and the anatomic basis of MJD/SCA3 disease. The volumetric 
Table 2. Correlations between volume structures and several parameters related to disease.

\begin{tabular}{llcc}
\hline Parameter & Structure & Sperman & p value \\
\hline Disease duration & L hemisphere & -0.7 & 0.04 \\
& Total cerebellum & -0.7 & 0.04 \\
& V2 & -0.69 & 0.005 \\
& R hemisphere & -0.56 & 0.02 \\
& Mesencephalon & -0.52 & 0.044 \\
CAG number & Total Vermis & -0.7 & 0.04 \\
& V1 & -0.69 & 0.004 \\
Age & Mesencephalon & -0.53 & 0.042 \\
& Total vermis & -0.78 & 0.01 \\
& L hemisphere & -0.73 & 0.002 \\
& R hemisphere & -0.71 & 0.003 \\
& Total cerebellum & -0.71 & 0.03 \\
& V1 & -0.68 & 0.004 \\
& V3 & -0.68 & 0.05 \\
V2 & -0.58 & 0.022 \\
& Pons & -0.68 & 0.01 \\
& L hemisphere & -0.63 & 0.02 \\
& Total brain stem & -0.62 & 0.01 \\
& Total cerebellum & -0.6 & 0.02 \\
& R hemisphere & -0.49 & 0.03 \\
V2 & -0.52 & 0.05 \\
Disease duration & 0.53 & 0.04 \\
\hline
\end{tabular}

method applied in the present study has some advantages due to the use of high resolution whole brain images, a robust algorithm for partial volume reduction, and normalization with ICV to avoid body volume and technical influence. These particularities may explain some of the differences we found ${ }^{17}$. In agreement to our data, reduced brainstem and cerebellar volume in MJD/ SCA3 patients has been widely demonstrated ${ }^{9-13}$. The most affected brain stem structure was the pons, with an average volume reduction of $35.7 \%$. This finding contrast with previous findings reporting only a mild pontine atrophy ${ }^{13,14}$. It is noteworthy that our measurements included part of the cerebellar peduncles and not only the ventral pons, since it is impossible to define the cerebellar anterior limit and brain stem anatomically. It has already been demonstrated that the volumes of the peduncles are decreased in the disease and that there is a positive correlation between the width of the cerebellar peduncle and the diameter of the pons ${ }^{12}$.

The most affected vermis subdivisions were V1 and $\mathrm{V} 2$ (with an average reduction of 37\%) whereas V3 was relatively spared with an average reduction of $24 \%$. Cerebellar hemispheres evidenced an average reduction of 19\%. Subdivisions of the vermis have not been studied previously; neither correlations between them and other parameters such as age, CAG number, disease duration and ICARS. According to our data, the disease process did not affect tonsils. Given tonsils are structures which prove difficult to limit, having partial volume effect; we used some of the strategies previously described - sagittal slices in parallel with coronal slices - to increase the accuracy of the measurements ${ }^{18}$.

\section{Disease duration}

We found an inverse correlation between disease duration and midbrain, V2, hemispheres and total cerebellum, but not with pons. This could be a time-related effect on the structures derived from midbrain, cerebellum and anterior vermis. Our results contrast with previous findings such as a negative correlation between disease duration and the pons area in MJD/SCA3 patients ${ }^{9}$. Nevertheless, one study failed to demonstrate a progression of midbrain and pontine tegmentum atrophy by aging or disease duration ${ }^{19}$. This study also corroborate with another one that describe a differential vulnerability in pontine structures: pontine tegmentum atrophy begins from the early stage of the disease and pontine base progressed negatively in correlation with disease duration ${ }^{9}$. In a more recent study, however, volumetry correlated $(p<0.10)$ with total brainstem, midbrain and putamen ${ }^{20}$, showing a diversity of findings in disease duration and atrophy in MJD/SCA3.

\section{CAG number}

We identified correlation among V1, midbrain and total vermis, with the number of CAG expansion. One study failed to demonstrate a correlation between CAG and brainstem or cerebellar volume ${ }^{13}$. They have suggested that the mutation per se, was responsible for atrophy and not the CAG number. One study showed a weak correlation between CAG number and midbrain, total brainstem, caudate nucleus and putamen ${ }^{20}$. Based on previous studies ${ }^{21-23}$ some authors, showed correlation between CAG and volume of tegmentum of pons divided by age ${ }^{9}$. We weren't able to find such correlation.

\section{Age}

Age and sex are the significant variables in volume measurement. Age had separate inverse correlation with all cerebellar structures and each of those of cerebellum, the cerebellar vermis being the most affected. According to some authors, brain stem structures remain unaltered regardless of disease duration ${ }^{21}$. Dorsum-medial areas (declive, folim and tuber-V2) and posterior lobe (pyramid, tonsil and nodule-V3) showed shrinking with age $^{18}$. It has been suggested that more recent phylogenetic areas, with several cortical extensions, are also susceptible to the aging effect ${ }^{22}$. To diminish bias, patients 
and controls were paired for age and sex. Structure volumes of controls didn't correlate with age. Such findings suggest that the neurodegenerative process in MJD/ SCA3 is also influenced by the patient age ${ }^{8}$.

\section{ICARS}

There was positive correlation between disease duration and ICARS, suggesting the progressive character of the disease. Pons, V2, total brain stem, hemispheres and total cerebellum correlated with ICARS. ICARS seems to be a valuable clinical parameter since it has been demonstrated a relevant inverse correlation between this scale and cerebellar atrophy ${ }^{24}$. An inverse correlation was found between ICARS and spinal cord area in MJD/SCA3 patients although with no statistical significance ${ }^{25}$. A previous study showed correlation among brainstem and cerebellar volumes and a clinical scale in SCA1 patients ${ }^{26}$. They also demonstrated a correlation between the volume of cerebellum and the duration of the disease in SCA1 and SCA2 patients. A more recent published study showed an inverse correlation between another clinical scale (Scale for the Assessment and Rating of Ataxia, SARA) and total brainstem and all substructures, caudate nucleus, total cerebellum and hemispheres. To our knowledge, there are only a few studies concerning volume measurements in SCAs and clinical data (specially with ICARS), while none have been conducted on MJD.

However, another study failed to demonstrate a correlation between pons volume and severity of illness and time of disease in SCA7 patients ${ }^{14}$. Conversely, they demonstrated a cerebellar correlation with both disease duration and severity of illness in SCA7 patients.

V2, hemispheres and total cerebellum volume also correlated with three variables: disease duration, disease severity (ICARS) and age. These correlations have never previously been demonstrated, and suggest these variables might play an important role in the disease process.

In conclusion, the most affected structure in this group of MJD/SCA3 patients was the pons, followed by cerebellar vermis and cerebellar hemispheres. Vermis and total cerebellum were the most sensitive structure related to age while brain stem and pons strongly correlated with disease severity (ICARS). Cerebellum and brain stem atrophy volumes measurement could be useful as a index in detecting neuronal vulnerability patterns in this disease, what may be useful in to indentify the progression of the disease and also as a parameter to be considered in future trials in MJD/SCA3.

\section{REFERENCES}

1. Silveira I, Coutinho P, Maciel P, et al. Analysis of SCA1, DRPLA, MJD, SCA2, and SCA6 CAG repeats in 48 Portuguese ataxia families. Am J Med Genet 1998;81:134-138.
2. Silveira I, Lopes-Cendes I, Kish S, et al. Frequency of spinocerebellar ataxia type 1, dentatorubropallidoluysian atrophy, and Machado-Joseph disease mutations in a large group of spinocerebellar ataxia patients. Neurology 1996;46:214-218.

3. Sasaki H, Yabe I, Tashiro K. The hereditary spinocerebellar ataxias in Japan Cytogenet Genome Res 2003;100:198-205.

4. Giunti P, Sabbadini G, Sweeney MG, et al. The role of the SCA2 trinucleotide repeat expansion in 89 autosomal dominant cerebellar ataxia families: frequency, clinical and genetic correlates. Brain 1998;121:459-467.

5. Soong BW, Lu YC, Choo KB, Lee HY. Frequency analysis of autosomal dominant cerebellar ataxias in Taiwanese patients and clinical and molecular characterization of spinocerebellar ataxia type 6. Arch Neurol 2001;58: 1105-1109.

6. Kawaguchi Y, Okamoto T, Taniwaki M, et al. CAG expansions in a novel gene for Machado-Joseph disease at chromosome 14q32.1. Nat Genet 1994;8:221-228

7. Nakano KK, Dawson DM, Spence A. Machado disease: a hereditary ataxia in Portuguese emigrants to Massachusetts. Neurology 1972;22:49-55.

8. Onodera $\mathrm{O}$, Idezuka J, Igarashi $\mathrm{S}$, et al. Progressive atrophy of cerebellum and brainstem as a function of age and the size of the expanded CAG repeats in the MJD1 gene in Machado-Joseph disease. Ann Neurol 1998;43: 288-296.

9. Yoshizawa, T, Watanabe M, Frusho K, Shoji S. Magnetic resonance imaging demonstrates differential atrophy of pontine base and tegmentum in Machado-Joseph disease. J Neurol Sci 2003;215:45- 50.

10. Lopes-Cendes I, Silveira I, Maciel P, et al. Limits of clinical assessment in the accurate diagnosis of Machado-Joseph disease. Arch Neurol 1996;53: 1168-1174.

11. Burk K, Abele M, Fetter M, et al. Autosomal dominant cerebellar ataxia type I clinical features and MRI in families with SCA1, SCA2 and SCA3. Brain 1996; 119:1497-1505.

12. Murata $\mathrm{Y}$, Yamaguchi S, Kawakami H, et al. Characteristic magnetic resonance imaging findings in Machado-Joseph disease. Arch Neurol 1998;55:33-37

13. Klockgether T, Skalej M, Wedekind D, et al. Autosomal dominant cerebellar ataxia type I: MRI-based volumetry of posterior fossa structures and basal ganglia in spinocerebellar ataxia types 1, 2 and 3. Brain 1998;121:1687-1693.

14. Bang OY, Lee PH, Kim SY, Kim HJ, Huh K. Pontine atrophy precedes cerebellar degeneration in spinocerebellar ataxia 7: MRI-based volumetric analysis. J Neurol Neurosurg Psychiatry 2004;75:1452-1456.

15. Trouillas $P$, Takayanagi T, Hallett M, et al. International Cooperative Ataxia Rating Scale for pharmacological assessment of the cerebellar syndrome: The Ataxia Neuropharmacology Committee of the World Federation of Neurology. J Neurol Sci 1997;145:205-211.

16. Luft AR, Skalej M, Welte D, et al. A new semiautomated, three-dimensional technique allowing precise quantification of total and regional cerebellar volume using MRI. Magn Resonan Med 1998;40:143-151.

17. Eritaia J, Wood SJ, Stuart GW, et al. An optimized method for estimating intracranial volume from magnetic resonance images. Magn Resonan Med 2000; 44:973-977.

18. Raz N, Dupuis JH, Briggs SD, McGavran C, Acker JD. Differential effects of age and sex on the cerebellar hemispheres and the vermis: a prospective MR study. AJNR Am J Neuroradiol 1998;19:65-71.

19. Horimoto $Y$, Matsumoto M, Yuasa H, et al. Brainstem in Machado-Joseph disease: atrophy or small size? Eur J Neurol 2008;15:102-105.

20. Schulz JB, Borkert J, Wolf S, et al. Visualization, quantification and correlation of brain atrophy with clinical symptoms in spinocerebellar ataxia types 1, 3 and 6. Neuroimage 2010;49:158-168.

21. Luft AR, Skalej M, Schulz JB, et al. Patterns of age-related shrinkage in cerebellum and brainstem observed in vivo using three-dimensional MRI volumetry. Cereb Cortex 1999;9:712-721.

22. Raz N, Torres IJ, Spencer WD, White K, Acker JD. Age-related regional differences in cerebellar vermis observed in vivo. Arch Neurol 1992;49:412-416.

23. Abe $Y$, Tanaka F, Matsumoto M, et al. CAG repeat number correlates with the rate of brainstem and cerebellar atrophy in Machado-Joseph disease. Neurology 1998;51:882-884.

24. Richter S, Dimitrova A, Maschke M, et al. Degree of cerebellar ataxia correlates with three-dimensional mri-based cerebellar volume in pure cerebellar degeneration. Eur Neurol 2005;54:23-27.

25. Lukas C, Hahn HK, Bellenberg B, et al. Spinal cord atrophy in spinocerebellar ataxia type 3 and 6: impact on clinical disability. J Neurol 2008;255: 1244-1249.

26. Guerrini L, Lolli F, Ginestroni A, et al. Brainstem neurodegeneration correlates with clinical dysfunction in SCA1 but not in SCA2: a quantitative volumetric, diffusion and proton spectroscopy MR study. Brain 2004;127: 1785-1795. 